\title{
Successful Kidney Transplant Despite Microthrombi From a Deceased Donor With Heparin Induced Thrombocytopenia
}

\author{
Adena J Osband ${ }^{\mathrm{a}, \mathrm{b}}$, Billie Fyfe ${ }^{\mathrm{a}}$, David A Laskow ${ }^{\mathrm{a}}$
}

\begin{abstract}
Many deceased donor factors determine utility of procured organs. In addition to the large dose of intravenous heparin usually given prior to aortic cross-clamp, kidneys are flushed with heparinized solution pre-implantation. In a donor with heparin induced thrombocytopenia (HIT), these exposures may lead to microthrombi, which may be a reason to refuse a kidney offer. We present a case of successful renal transplantation of a highly sensitized patient from a donor with HIT and microthrombi on biopsy.
\end{abstract}

Keywords: Kidney transplant; Microthrombi; Deceased donor

\section{Introduction}

There are scattered references in the literature to deceased donors receiving heparin substitutes in the case of known heparin induced thrombocytopenia (HIT) [1-3]. It is also clear that microthrombi on a pre-implantation biopsy may be a reason to refuse a kidney offer [4]. We present here a case where a deceased donor received heparin despite HIT, and the kidney was transplanted with good results despite microthrombi on biopsy.

\section{Case Report}

The donor was a 58-year-old woman who died of thrombotic

Manuscript accepted for publication December 3, 2013

\footnotetext{
${ }^{a}$ Rutgers/Robert Wood Johnson Medical School, New Brunswick, NJ 08901, USA

${ }^{\mathrm{b}}$ Corresponding author: Adena J Osband, Kidney/Pancreas Transplant, 10 Plum St, 7th floor, New Brunswick, NJ 08901, USA.

Email: adena.osband@rwjuh.edu
}

doi: http://dx.doi.org/10.14740/jcs209w complications from established HIT (extensive aortic thrombus, femoral \& pulmonary emboli, \& cerebral infarct). Per local procurement protocol, she received 30,000 units heparin intravenously prior to aortic cross-clamp and perfusion. Frozen section of the right kidney demonstrated a single arterial thrombus. Permanent section (Fig. 1) later revealed multiple arterial and glomerular capillary thrombi.

The recipient is a 70 -year-old woman with renal failure due to focal and segmental glomerulosclerosis, on dialysis for 5 years, with negative cytotoxic and flow crossmatches. Because of her sensitization history $(100 \%$ panel reactive antibody, 47 unacceptable antigens), she received plasmapheresis and intravenous immunoglobulin in addition to routine induction immunosuppression. Also in view of her sensitization history, we accepted this donor kidney despite the thrombus. The kidney worked immediately, and transplant function at two years is excellent (creatinine $1.3 \mathrm{mg} / \mathrm{dL}$ ).

\section{Discussion}

Donors with HIT are not a new consideration in the realm of organ procurement. No trials have evaluated the use of heparin compared to alternates in deceased donors, but there are scattered case reports in the literature investigating effects on the transplanted organs. One paper details use of organs

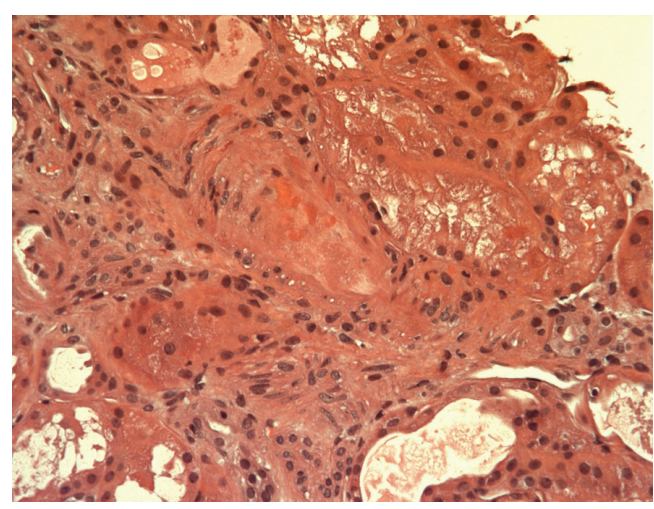

Figure 1. Permanent section of pre-implantation kidney biopsy demonstrating glomerular capillary and arterial thrombi. 
from donors who received argatroban which were successfully transplanted without complications [1]. A second case reports re-use of a liver allograft from an original recipient who developed HIT and received lepirudin at procurement, with no noted sequelae in the second recipient [2]. Yet another case details a significant coagulapathy in a liver recipient, attributed to the large argatroban bolus the donor received, but no coagulopathies in the other organ recipients from that donor [3].

An abstract presented in 1978 which demonstrated a 33\% graft failure rate in kidneys with microthrombi resulted in many transplant centers avoiding kidneys with microthrombi on biopsy [5]. Because this is so universally accepted, it is not well detailed in the literature. A few small studies detail use of kidneys from donors with disseminated intravascular coagulation (DIC) and glomerular microthrombi, reporting no impact on graft function [6-8]. One center treated kidneys with microthrombi with tPA prior to implantation and yielded initial good results [9]. Another case report describes successful use of the kidneys from a donor with DIC associated with self-limited initial DIC in both recipients [10].

In conclusion, consideration should be given to use of heparin alternates during procurement in HIT donors, although which is preferable or the ideal dose have yet to be determined. In addition, presence of microthrombi on renal biopsy may not be a contraindication to transplantation, especially for sensitized recipients.

\section{References}

1. Aloia TA, Goss JA. A report of outcomes after orthotopic liver transplant with allografts from heparin antibodypositive donors. Exp Clin Transplant. 2009;7(1):13-17.

2. Rentsch M, Meyer J, Andrassy J, Fischer-Frohlich CL, Rust C, Mueller S, Angele M, et al. Late reuse of liver allografts from brain-dead graft recipients: the Munich experience and a review of the literature. Liver Transpl. 2010;16(6):701-704.

3. Schwartz JJ, Hatch JM, Book Z, Lonardo N, Hutson WR, Sorensen JB. Use of argatroban during multi-organ procurement: pharmacokinetics and sequelae in recipient of transplanted liver. Clin Transplant. 2009;23(5):705-709.

4. Farney AC, Rogers J, Orlando G, al-Geizawi S, Buckley M, Farooq U, al-Shraideh Y, et al. Evolving experience using kidneys from deceased donors with terminal acute kidney injury. J Am Coll Surg. 2013;216(4):645-655; discussion 655-646.

5. Meyers AM, Lewin J, Disler PB, et al. Donor disseminated intravascular coagulation (DIC), intraglomerular fibrin deposition, and subsequent graft function. Kidney International. 1978;13:432.

6. Pastural M, Barrou B, Delcourt A, Bitker MO, Ourahma S, Richard F. Successful kidney transplantation using organs from a donor with disseminated intravascular coagulation and impaired renal function: case report and review of the literature. Nephrol Dial Transplant. 2001;16(2):412-415.

7. McCall SJ, Tuttle-Newhall JE, Howell DN, Fields TA. Prognostic significance of microvascular thrombosis in donor kidney allograft biopsies. Transplantation. 2003;75(11):1847-1852.

8. Wang CJ, Shafique S, McCullagh J, Diederich DA, Winklhofer FT, Wetmore JB. Implications of donor disseminated intravascular coagulation on kidney allograft recipients. Clin J Am Soc Nephrol. 2011;6(5):1160-1167.

9. Nghiem DD, Olson PR, Sureshkumar KK. Role of pulsatile perfusion with tissue plasminogen activator in deceased donor kidneys with extensive glomerular thrombosis. Transplant Proc. 2009;41(1):29-31.

10. Bennett WM, Hansen KS, Houghton DC, McEvoy KM. Disseminated intravascular coagulation (DIC) in a kidney donor associated with transient recipient DIC. Am J Transplant. 2005;5(2):412-414. 